\title{
RELATIVE $\mathbb{Q}$-GRADINGS FROM BORDERED FLOER THEORY
}

\author{
ROBERT LIPSHITZ, PETER OZSVÁTH, AND DYLAN P. THURSTON
}

\begin{abstract}
In this paper we show how to recover the relative $\mathbb{Q}$-grading in Heegaard Floer homology from the noncommutative grading on bordered Floer homology.
\end{abstract}

\section{CONTEnTs}

1. Introduction 1

2. Background 2

2.1. The relative $\mathbb{Q}$-grading 2

2.2. The structure of bordered Floer theory 3

2.3. The (non-commutative) grading in bordered Floer 4

3. From bordered Floer to the relative $\mathbb{Q}$-grading 7

4. Examples 9

$\begin{array}{ll}\text { References } & 10\end{array}$

\section{INTRODUCTION}

Heegaard Floer homology, introduced by the second author and Z. Szabó, is an invariant for a three-manifold equipped with a $\operatorname{spin}^{c}$ structure [OSz04]. Heegaard Floer homology is defined as Lagrangian intersection Floer homology groups of certain Lagrangians in a symmetric product of a Riemann surface, and as such is most naturally are only relatively, cyclicly graded. Indeed, the Heegaard Floer homology of a three-manifold equipped with the $\operatorname{spin}^{c}$ structure $\mathfrak{s}$ is graded by the group $\mathbb{Z} / \operatorname{div}\left(c_{1}(\mathfrak{s})\right)$, where $\operatorname{div}\left(c_{1}(\mathfrak{s})\right)$ denotes the divisibility of the first Chern class of the $\operatorname{spin}^{c}$ structure $\mathfrak{s}$. In particular, if the first Chern class of $\mathfrak{s}$ is torsion, then the corresponding Heegaard Floer homology is relatively $\mathbb{Z}$-graded.

With the help of the functorality properties of Heegaard Floer homology, the relative $\mathbb{Z}$-grading on Heegaard Floer homology can be lifted to an absolute $\mathbb{Q}$-grading when the underlying $\operatorname{spin}^{c}$ structure is torsion [OSz06]. (Compare Frøyshov [Frø04].) This absolute $\mathbb{Q}$-grading contains subtle topological information; for a beautiful recent application, see [Gre]. Although, by work of Sarkar-Wang [SW10] and Sarkar [Sar10], the absolute $\mathbb{Q}$ grading is algorithmically computable, no simple formula is known, and it remains somewhat mysterious.

By contrast, the relative $\mathbb{Q}$-grading induced by the absolute $\mathbb{Q}$-grading is much simpler. In this paper, we show how to use bordered Floer homology to compute this relative $\mathbb{Q}$-grading between different torsion $\operatorname{spin}^{c}$ structures, by decomposing a 3-manifold along a connected surface; it turns out that the non-commutative grading on bordered Floer homology contains

RL was supported by an NSF Grant DMS-0905796 and a Sloan Research Fellowship.

PSO was supported by NSF grant number DMS-0804121.

DPT was supported by NSF grant number DMS-1008049 and a Sloan Research Fellowship. 
the necessary information. (Another way of computing the relative $\mathbb{Q}$-grading, using covering spaces, was given by D. Lee and the first author [LL08].)

Finally, note that Heegaard Floer homology has several variants, $\widehat{H F}, H F^{-}, H F^{\infty}$, and $H F^{+}$. Although we focus on the relative $\mathbb{Q}$-grading on $\widehat{H F}$ (as that is the version with a corresponding bordered theory), this determines the relative $\mathbb{Q}$-grading on $\mathrm{HF}^{+}, \mathrm{HF}^{-}$and $H F^{\infty}$, via the exact triangles

$$
\cdots \longrightarrow \widehat{H F}(Y) \longrightarrow H F^{+}(Y) \stackrel{\cdot U}{\longrightarrow} H F^{+}(Y) \stackrel{[1]}{\longrightarrow} \cdots
$$

and

$$
\cdots \longrightarrow H F^{-}(Y) \longrightarrow H F^{\infty}(Y) \longrightarrow H F^{+}(Y) \stackrel{[1]}{\longrightarrow} \cdots .
$$

Acknowledgements. This paper was started while we were visiting the Mathematical Sciences Research Institute (MSRI) and finished while the first two authors were visiting the Simons Center for Geometry and Physics and the third author was visiting U. C. Berkeley and MSRI. We thank all three institutions for their hospitality.

\section{BACKGROUND}

2.1. The relative $\mathbb{Q}$-grading. The absolute $\mathbb{Q}$-grading on $\widehat{H F}(Y, \mathfrak{s})$ is defined as follows. Choose a $\operatorname{spin}^{c}$ nullcobordism $\left(W^{4}, \mathfrak{t}\right)$ of $(Y, \mathfrak{s})$. Associated to the cobordism $W$ is a map $\hat{F}_{W, \mathfrak{s}}: \widehat{H F}\left(S^{3}, \mathfrak{s}_{0}\right) \rightarrow \widehat{H F}(Y, \mathfrak{s})$. The absolute grading on $\widehat{H F}(Y, \mathfrak{s})$ is characterized by the property that the generator of $\widehat{H F}\left(S^{3}\right) \cong \mathbb{Z}$ lies in degree 0 and the map $F$ has degree

$$
\frac{c_{1}(\mathfrak{t})^{2}-2 \chi(W)-3 \sigma(W)}{4} .
$$

(Actually, since $\hat{F}_{W, \mathfrak{s}}$ might be trivial on homology, it is more accurate to say the grading is characterized by the property that Maslov index 0 triangles in the definition of $\hat{F}_{W, \mathfrak{s}}$ have this degree.) See [OSz06, Section 7].

The paper [LL08] shows that the relative $\mathbb{Q}$-grading can be reformulated as follows. Suppose that $\mathbf{x}$ and $\mathbf{y}$ are generators for $\widehat{C F}(Y)$ (computed via some pointed Heegaard diagram $\mathcal{H}=(\Sigma, \boldsymbol{\alpha}, \boldsymbol{\beta}, z))$ so that $c_{1}(\mathfrak{s}(\mathbf{x}))$ and $c_{1}(\mathfrak{s}(\mathbf{y}))$ are torsion. Then there is a finite-order covering space $p: \widetilde{Y} \rightarrow Y$ so that $p^{*} \mathfrak{s}(\mathbf{x})=p^{*} \mathfrak{s}(\mathbf{y})$ [LL08, Corollary 2.10]. The Heegaard diagram $\mathcal{H}$ for $Y$ lifts to a (multi-pointed) Heegaard diagram $\widetilde{\mathcal{H}}$ for $\widetilde{Y}$. The generators $\mathbf{x}$ and $\mathbf{y}$ have preimages $p^{-1}(\mathbf{x})$ and $p^{-1}(\mathbf{y})$ in $\widetilde{\mathcal{H}}$ so that $\mathfrak{s}\left(p^{-1}(\mathbf{x})\right)=p^{*} \mathfrak{s}(\mathbf{x})$ and $\mathfrak{s}\left(p^{-1}(\mathbf{y})\right)=p^{*} \mathfrak{s}(\mathbf{y})$. Thus, $p^{-1}(\mathbf{x})$ and $p^{-1}(\mathbf{y})$ have a well-defined $\mathbb{Z}$-grading difference. Then

$$
\operatorname{gr}_{\mathbb{Q}}(\mathbf{y})-\operatorname{gr}_{\mathbb{Q}}(\mathbf{x})=\frac{1}{n} \operatorname{gr}_{\mathbb{Z}}\left(p^{*}(\mathbf{x}), p^{*}(\mathbf{y})\right)
$$

where $n$ is the order of the cover $\widetilde{Y} \rightarrow Y$.

More concretely, even though $\mathbf{x}$ and $\mathbf{y}$ can not necessarily be connected by a domain in $\pi_{2}(\mathbf{x}, \mathbf{y})$, if we allow rational multiples of the regions in $\Sigma$ then they can be connected. That is, let $\pi_{2}^{\mathbb{Q}}(\mathbf{x}, \mathbf{y})$ denote the set of rational linear combinations $B$ of components of $\Sigma \backslash(\boldsymbol{\alpha} \cup \boldsymbol{\beta})$ connecting $\mathbf{x}$ to $\mathbf{y}$ (i.e., $\partial(\partial B \cap \boldsymbol{\alpha})=-\partial(\partial B \cap \boldsymbol{\beta})=\mathbf{y}-\mathbf{x})$. If $c_{1}(\mathfrak{s}(\mathbf{x}))-c_{1}(\mathfrak{s}(\mathbf{y}))$ is torsion then $\pi_{2}^{\mathbb{Q}}(\mathbf{x}, \mathbf{y})$ is nonempty, and

$$
\operatorname{gr}_{\mathbb{Q}}(\mathbf{y})-\operatorname{gr}_{\mathbb{Q}}(\mathbf{x})=e(B)+n_{\mathbf{x}}(B)+n_{\mathbf{y}}(B)
$$


for any $B \in \pi_{2}^{\mathbb{Q}}(\mathbf{x}, \mathbf{y})$. Here, $e(B)$ denotes the Euler measure of $B$ and $n_{\mathbf{x}}(B)$ and $n_{\mathbf{y}}(B)$ denote the point measure of $B$ at the points $\mathbf{x}$ and $\mathbf{y}$; compare [Lip06, Section 4.2]. These quantities on the right are thought of as the natural $\mathbb{Q}$-linear extensions of the usual integervalued analogues. This agrees with the formulas in [LL08, Section 2.3].

2.2. The structure of bordered Floer theory. Bordered Floer theory assigns to a surface $F=F(\mathcal{Z})$ represented by a pointed matched circle $\mathcal{Z}$ (Figure 1) a $d g$ algebra $\mathcal{A}=\mathcal{A}(\mathcal{Z})$ [LOT08, Section 3]. To a 3 -manifold $Y$ with boundary parameterized by $F(\mathcal{Z})$ it associates invariants $\widehat{C F A}(Y)_{\mathcal{A}(\mathcal{Z})}$, a right $\mathcal{A}_{\infty}$-module over $\mathcal{A}(\mathcal{Z})$, and ${ }^{\mathcal{A}(-\mathcal{Z})} \widehat{C F D}(Y)$, a left, projective $d g$ module over $\mathcal{A}(-\mathcal{Z})$ [LOT08, Sections 6, 7]. Each of $\widehat{C F A}(Y)$ and $\widehat{C F D}(Y)$ are well-defined up to homotopy equivalence. These modules are related to the invariants of a closed 3-manifold by a pairing theorem:

Theorem 2.1. [LOT08, Theorem 1.3] If $Y_{1}$ and $Y_{2}$ are 3-manifolds with boundaries parameterized by $F(\mathcal{Z})$ and $-F(\mathcal{Z})$ respectively then

$$
\widehat{C F}\left(Y_{1} \cup_{F} Y_{2}\right) \simeq \widehat{C F A}\left(Y_{1}\right) \widetilde{\otimes}_{\mathcal{A}(\mathcal{Z})} \widehat{C F D}\left(Y_{2}\right) \text {. }
$$

Here, $\widetilde{\otimes}_{\mathcal{A}(\mathcal{Z})}$ denotes the $\mathcal{A}_{\infty}$-tensor product over $\mathcal{A}(\mathcal{Z})$. There is a particularly convenient model $\otimes$ for the $\mathcal{A}_{\infty}$-tensor product so that $\widehat{C F}\left(Y_{1} \cup_{F} Y_{2}\right)$ is actually isomorphic as an $\mathbb{F}_{2^{-}}$ vector space to $\widehat{C F A}\left(Y_{1}\right) \otimes \widehat{C F D}\left(Y_{2}\right)$ (for corresponding choices of auxiliary data, as discussed below).

The isomorphism in Theorem 2.1 is an isomorphism of relatively graded groups, in an appropriate sense. This will be discussed further in Section 2.3.

For the purposes of this paper, we will use the following basic facts about $\mathcal{A}(\mathcal{Z}), \widehat{C F A}(Y)$ and $\widehat{C F D}(Y)$ :

- The invariants $\widehat{C F A}(Y)$ and $\widehat{C F D}(Y)$ are defined in terms of a bordered Heegaard diagram $\mathcal{H}=(\Sigma, \boldsymbol{\alpha}, \boldsymbol{\beta}, z)$ for $Y$. Here, $\Sigma$ is a compact, orientable surface of some genus $g$ with one boundary component; $\boldsymbol{\alpha}$ consists of pairwise-disjoint embedded arcs $\boldsymbol{\alpha}^{a}$ and circles $\boldsymbol{\alpha}^{c}$ in $\Sigma$, with $\partial \boldsymbol{\alpha}^{a} \subset \partial \Sigma$, while $\boldsymbol{\beta}$ consists of embedded circles only; and $z$ is a basepoint in $\partial \Sigma$, not lying on any $\alpha$-arc. See Figure 2 for an example, and [LOT08, Section 4] for more details.

- If the bordered Heegaard diagrams $\mathcal{H}_{1}$ and $H_{2}$ represent $Y_{1}$ and $Y_{2}$, respectively, and $\partial Y_{1}=F(\mathcal{Z})=-\partial Y_{2}$, then $\mathcal{H}=\mathcal{H}_{1} \cup_{\partial} \mathcal{H}_{2}$ represents $Y=Y_{1} \cup_{\partial} Y_{2}$.

- Given a genus $g$ bordered Heegaard diagram $\mathcal{H}$ representing $Y$, the modules $\widehat{C F D}(Y)$ and $\widehat{C F A}(Y)$ are generated by all sets $\mathbf{x}=\left\{x_{1}, \ldots, x_{g}\right\}$ of $g$ points in $\boldsymbol{\alpha} \cap \boldsymbol{\beta}$ so that exactly one $x_{i}$ lies on each $\alpha$ - or $\beta$-circle and at most one $x_{i}$ lies on each $\alpha$-arc. (Again, see Figure 2.) Let $\mathfrak{S}(\mathcal{H})$ denote the set of generators $\mathbf{x}$ in $\mathcal{H}$.

- Given generators $\mathbf{x}$ and $\mathbf{y}$ in $\mathfrak{S}(\mathcal{H})$, a domain connecting $\mathbf{x}$ to $\mathbf{y}$ is a linear combination $B$ of components of $\Sigma \backslash(\boldsymbol{\alpha} \cup \boldsymbol{\beta})$ so that $\partial((\partial B) \cap \boldsymbol{\beta})=\mathbf{x}-\mathbf{y}$ and $\partial((\partial B) \cap(\boldsymbol{\alpha} \cup \partial \Sigma))=$ $\mathbf{y}-\mathbf{x}$. (See Figure 2.) Let $\pi_{2}(\mathbf{x}, \mathbf{y})$ denote the set of domains connecting $\mathbf{x}$ to $\mathbf{y}$. For $B \in \pi_{2}(\mathbf{x}, \mathbf{y})$, let $\partial^{\alpha} B=(\partial B) \cap \boldsymbol{\alpha}, \partial^{\beta} B=(\partial B) \cap \boldsymbol{\beta}$ and $\partial^{\partial} B=(\partial B) \cap(\partial \Sigma)$.

- Given a bordered Heegaard diagram $\mathcal{H}$ for $Y$, associated to each generator $\mathbf{x} \in$ $\mathfrak{S}(\mathcal{H})$ is a $\operatorname{spin}^{c}$-structure $\mathfrak{s}(\mathbf{x})$ on $Y$. The modules $\widehat{C F D}(Y)$ and $\widehat{C F A}(Y)$ decompose according to these $\operatorname{spin}^{c}$-structures, $\widehat{C F D}(Y)=\bigoplus_{\mathfrak{s} \in \operatorname{spin}^{c}(Y)} \widehat{C F D}(Y ; \mathfrak{s})$ and $\widehat{C F A}(Y)=$ 

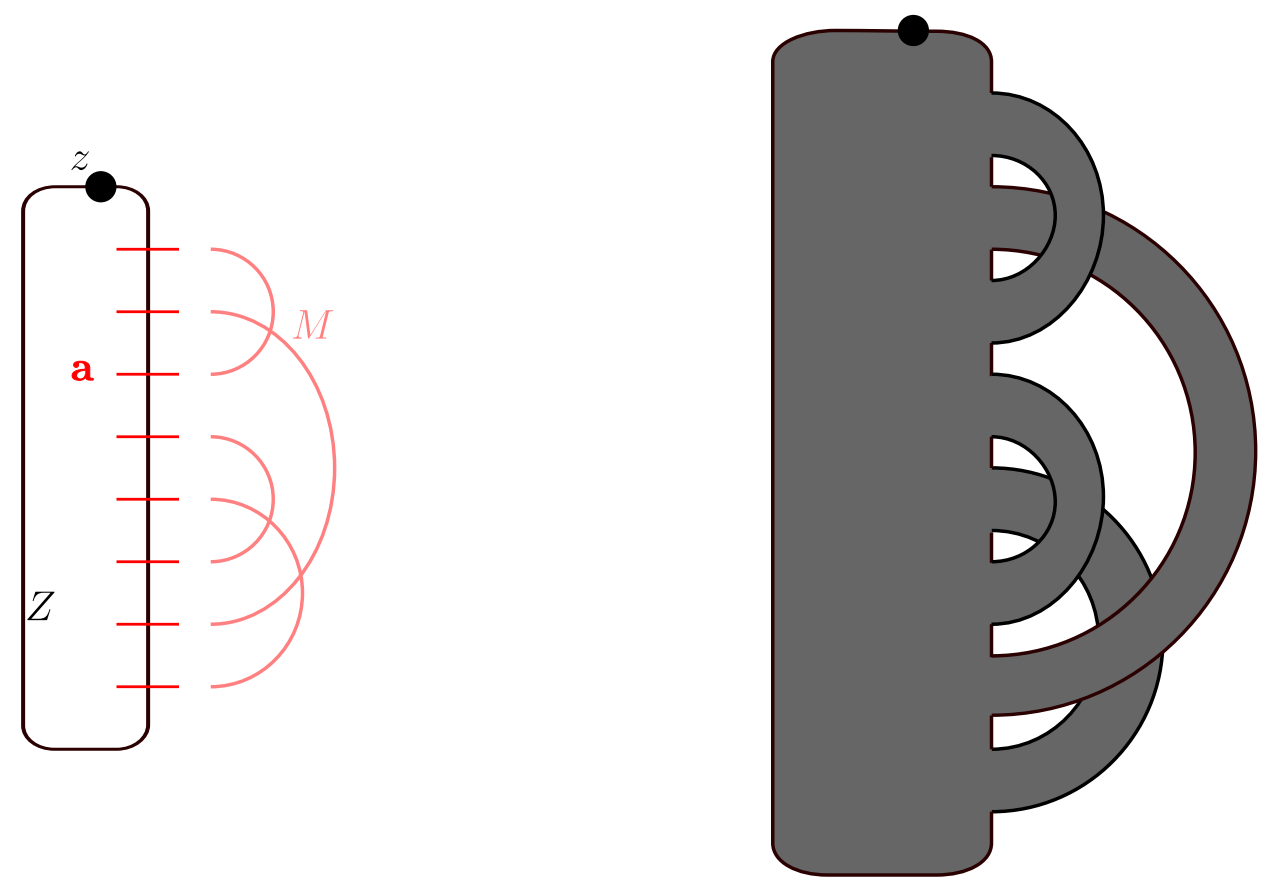

FigURE 1. The surface represented by a pointed matched circle. Left: a pointed matched circle $\mathcal{Z}$, consisting of a circle $Z, 4 k$ points a (the case $k=2$ is shown) matched in pairs via a matching $M$, and with a basepoint $z$. Right: the surface with boundary $F^{\circ}(\mathcal{Z})$ represented by $\mathcal{Z}$. The surface $F(\mathcal{Z})$ represented by $\mathcal{Z}$ is obtained by gluing a disk to $F^{\circ}(\mathcal{Z})$. As such, it contains a distinguished disk and a basepoint on the boundary of that disk.

$\bigoplus_{\mathfrak{s} \in \operatorname{spin}^{c}(Y)} \widehat{C F A}(Y ; \mathfrak{s})$. Let $\mathfrak{S}(\mathcal{H}, \mathfrak{s})=\{\mathbf{x} \in \mathfrak{S}(\mathcal{H}) \mid \mathfrak{s}(\mathbf{x})=\mathfrak{s}\}$ denote the set of generators for $\widehat{C F A}(Y, \mathfrak{s})$ and $\widehat{C F D}(Y, \mathfrak{s})$.

- Given bordered Heegaard diagrams $\mathcal{H}_{1}$ and $\mathcal{H}_{2}$ with $\partial \mathcal{H}_{1}=-\partial \mathcal{H}_{2}$, let $\mathcal{H}=\mathcal{H}_{1} \cup_{\partial} \mathcal{H}_{2}$. There is an obvious embedding $\mathfrak{S}(\mathcal{H}) \rightarrow \mathfrak{S}\left(\mathcal{H}_{1}\right) \times \mathfrak{S}\left(\mathcal{H}_{2}\right)$ of the set of generators $\mathfrak{S}(\mathcal{H})$ of $\widehat{C F}(\mathcal{H})$. The image of this embedding is the set of pairs $\left(\mathbf{x}_{1}, \mathbf{x}_{2}\right) \in \mathfrak{S}\left(\mathcal{H}_{1}\right) \times \mathfrak{S}\left(\mathcal{H}_{2}\right)$ so that $\mathbf{x}_{1}$ and $\mathbf{x}_{2}$ occupy complementary $\alpha$-arcs. It turns out that these are exactly the generators of $\widehat{C F A}\left(\mathcal{H}_{1}\right) \otimes \widehat{C F D}\left(\mathcal{H}_{2}\right)$.

There is an extension of bordered Floer theory to manifolds with two boundary components, which are assigned various types of bimodules [LOT10a]. The generalizations of the results of this paper to the bimodule case are straightforward, and we shall not discuss them.

2.3. The (non-commutative) grading in bordered Floer. As noted in the introduction, the grading on bordered Floer homology is non-commutative.

Definition 2.2. Let $G$ be a group and $\lambda$ a central element in $G$. If $\mathcal{A}$ is a differential algebra, a grading of $\mathcal{A}$ by $G$ consists of a decomposition (as abelian groups) $\mathcal{A}=\bigoplus_{g \in G} \mathcal{A}_{g}$ of $\mathcal{A}$ into 


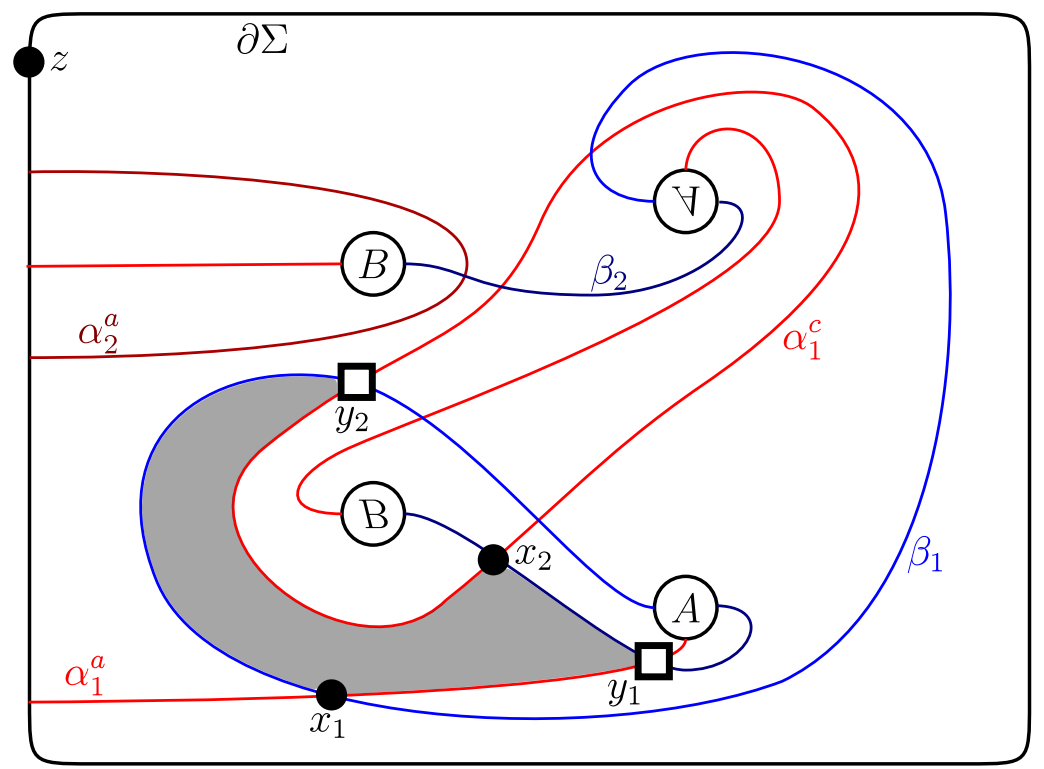

Figure 2. A bordered Heegaard diagram. The circles labeled $A$ and $B$ are connected by tubes. This bordered Heegaard diagram represents the trefoil complement (with a particular parameterization of its boundary). Two generators in $\mathfrak{S}(\mathcal{H})$ are marked, one by solid disks and the other by empty squares. A domain $B \in \pi_{2}(\mathbf{x}, \mathbf{y})$ connecting $\mathbf{x}$ and $\mathbf{y}$ is also shown, shaded.

homogeneous parts so that for any homogeneous algebra elements $a$ and $b$,

$$
\begin{array}{ll}
\operatorname{gr}(a b)=\operatorname{gr}(a) \operatorname{gr}(b) & \text { if } a b \neq 0 \\
\operatorname{gr}(\partial a)=\lambda^{-1} \operatorname{gr}(a) & \text { if } \partial a \neq 0 .
\end{array}
$$

Let $S$ be a left $G$-set. If ${ }_{\mathcal{A}} M$ is a left differential $\mathcal{A}$-module, a grading of ${ }_{\mathcal{A}} M$ by $S$ consists of a decomposition (as abelian groups) $M=\bigoplus_{s \in S} M_{s}$ of $M$ into homogeneous parts such that for homogeneous elements $x \in M$ and $a \in \mathcal{A}$,

$$
\begin{array}{ll}
\operatorname{gr}(a x)=\operatorname{gr}(a) \operatorname{gr}(x) & \text { if } a x \neq 0 \\
\operatorname{gr}(\partial x)=\lambda^{-1} \operatorname{gr}(x) & \text { if } \partial x \neq 0 .
\end{array}
$$

More generally, if $S$ is an $\mathcal{A}_{\infty}$-module over $\mathcal{A}$, Equations (2.5) and (2.6) become

$$
\operatorname{gr}\left(m_{k+1}\left(a_{1}, \ldots, a_{k}, x\right)\right)=\lambda^{k-1} \operatorname{gr}\left(a_{1}\right) \cdots \operatorname{gr}\left(a_{k}\right) \operatorname{gr}(x),
$$

if $m_{k+1}\left(a_{1}, \ldots, a_{k}, x\right) \neq 0$.

Gradings on right modules by right $G$-sets are defined similarly.

In the case of bordered Floer homology, for each pointed matched circle $\mathcal{Z}=(Z, \mathbf{a}, M, z)$ there is a group $G^{\prime}(\mathcal{Z})$ so that $\mathcal{A}(\mathcal{Z})$ is graded by $G^{\prime}(\mathcal{Z})$. The group $G^{\prime}(\mathcal{Z})$ is a $\mathbb{Z}$-central extension of $H_{1}(Z, \mathbf{a})$. To specify it, given a point $p \in \mathbf{a}$ and homology class $c \in H_{1}(Z, \mathbf{a})$, define $\mu(a, p)$ to be the average local multiplicity of $a$ near $p$. Extend $\mu$ to a bilinear map $H_{1}(Z, \mathbf{a}) \otimes H_{0}(\mathbf{a}) \rightarrow \frac{1}{2} \mathbb{Z}$. Then $G^{\prime}$ is defined by the commutation relation

$$
g \cdot h=\lambda^{2 \mu([h], \partial[g])} h \cdot g,
$$


where [.]: $G^{\prime}(\mathcal{Z}) \rightarrow H_{1}(Z, \mathbf{a})$ is the canonical projection and $\lambda$ is a generator of the central $\mathbb{Z}$.

Explicitly, we can write elements of $G^{\prime}(\mathcal{Z})$ as pairs $(m, c)$ where $m \in \mathbb{Q}$ and $c \in H_{1}(Z, \mathbf{a})$, with multiplication given by

$$
\left(m_{1}, \alpha_{1}\right) \cdot\left(m_{2}, \alpha_{2}\right)=\left(m_{1}+m_{2}+m\left(\alpha_{2}, \partial \alpha_{1}\right), \alpha_{1}+\alpha_{2}\right) .
$$

The group $G^{\prime}(\mathcal{Z})$ is generated by the elements $\lambda=(1 ; 0)$ and $\left(-\frac{1}{2},[i, i+1]\right)$.

The grading on $\widehat{C F A}(\mathcal{H}, \mathfrak{s})$ is given as follows. Given a Heegaard diagram $\mathcal{H}$ with $\partial \mathcal{H}=\mathcal{Z}$ and a $\operatorname{spin}^{c}$-structure $\mathfrak{s}$ on $Y=Y(\mathcal{H})$, fix a base generator $\mathbf{x}_{0} \in \mathfrak{S}(\mathcal{H}, \mathfrak{s})$. For a domain $B \in \pi_{2}(\mathbf{x}, \mathbf{y})$ define

$$
g^{\prime}(B)=\left(-e(B)-n_{\mathbf{x}}(B)-n_{\mathbf{y}}(B), \partial^{\partial} B\right) \in G^{\prime}(\mathcal{Z}) .
$$

If $B_{1} \in \pi_{2}(\mathbf{x}, \mathbf{y})$ and $B_{2} \in \pi_{2}(\mathbf{y}, \mathbf{z})$, let $B_{1} * B_{2} \in \pi_{2}(\mathbf{x}, \mathbf{z})$ denote the concatenation of $B_{1}$ and $B_{2}$. Then $g^{\prime}\left(B_{1} * B_{2}\right)=g^{\prime}\left(B_{1}\right) \cdot g^{\prime}\left(B_{2}\right)$ [LOT08, Lemma 6.15]. In particular, $P^{\prime}\left(\mathbf{x}_{0}\right)=$ $\left\{g^{\prime}(B) \mid B \in \pi_{2}\left(\mathbf{x}_{0}, \mathbf{x}_{0}\right)\right\}$ is a subgroup of $G^{\prime}(\mathcal{Z})$. The module $\widehat{C F A}(\mathcal{H}, \mathfrak{s})$ is graded by the right $G^{\prime}(\mathcal{Z})$-set $G_{A}^{\prime}(\mathcal{H}, \mathfrak{s}):=P^{\prime}\left(\mathbf{x}_{0}\right) \backslash G^{\prime}(4 k)$. (This construction depends on $\mathbf{x}_{0}$, but different choices of $\mathbf{x}_{0}$ give canonically isomorphic grading sets; see [LOT08, Section 10.3].) The grading of an element $\mathbf{x} \in \mathfrak{S}(\mathcal{H}, \mathfrak{s})$ is given by $g(B)$ for any $B \in \pi_{2}\left(\mathbf{x}_{0}, \mathbf{x}\right)$, thought of as an element of the coset space $G_{A}^{\prime}(\mathcal{H}, \mathfrak{s}):=P^{\prime}\left(\mathbf{x}_{0}\right) \backslash G^{\prime}(4 k)$.

The invariant $\widehat{C F D}(\mathcal{H})$ is a module over $\mathcal{A}(-\partial \mathcal{H})$ rather than $\partial \mathcal{H}$. So, in grading $\widehat{C F D}(\mathcal{H})$ we will use the anti-homomorphism $R: G^{\prime}(-\mathcal{Z}) \rightarrow G^{\prime}(\mathcal{Z})$ given by $R(j, \alpha)=\left(j, r_{*}(\alpha)\right)$ where $r:-Z \rightarrow Z$ is the (orientation-reversing) identity map. The grading on $\widehat{C F D}(\mathcal{H}, \mathfrak{s})$ is then defined similarly to the grading on $\widehat{C F A}(\mathcal{H}, \mathfrak{s})$, except that the left module $\widehat{C F D}(\mathcal{H}, \mathfrak{s})$ is graded by the left $G^{\prime}$-set $G_{D}^{\prime}(\mathcal{H}, \mathfrak{s}):=G^{\prime}(4 k) / R\left(P^{\prime}\left(\mathbf{x}_{0}\right)\right)$, and the grading of an element $x \in \mathfrak{S}(\mathcal{H}, \mathfrak{s})$ is given by (the equivalence class of) $R(g(B))$ for any $B \in \pi_{2}\left(\mathbf{x}_{0}, \mathbf{x}\right)$.

The tensor product $\widehat{C F A}\left(\mathcal{H}_{1}, \mathfrak{s}_{1}\right) \otimes \widehat{C F D}\left(\mathcal{H}_{2}, \mathfrak{s}_{2}\right)$ is graded by the amalgamated product of the grading sets $G_{A}^{\prime}\left(\mathcal{H}_{1}\right) \times_{G^{\prime}} G_{D}^{\prime}\left(\mathcal{H}_{2}\right)$; the grading of $\mathbf{x}_{1} \otimes \mathbf{x}_{2}$ is $\operatorname{gr}^{\prime}\left(\mathbf{x}_{1} \otimes \mathbf{x}_{2}\right)=\left(\operatorname{gr}^{\prime}\left(\mathbf{x}_{1}\right), \operatorname{gr}^{\prime}\left(\mathbf{x}_{2}\right)\right)$. (In fact, certain results are cleaner if one works instead with a certain subset of this amalgamated product that contains the gradings of all tensor products of generators; compare Theorem 2.9, below, and [LOT08, Theorem 10.43].) Note that since $\lambda$ is central in $G^{\prime}$, the set $G_{A}^{\prime}\left(\mathcal{H}_{1}\right) \times_{G^{\prime}} G_{D}^{\prime}\left(\mathcal{H}_{2}\right)$ retains an action by $\lambda$, which we will think of as a $\mathbb{Z}$-action.

A graded version of the pairing theorem states:

Theorem 2.9. [LOT08, Theorem 9.33] If $Y_{1}$ and $Y_{2}$ are 3-manifolds with boundaries parameterized by $F$ and $-F$ respectively then there is a map

$$
\Phi: \widehat{C F A}\left(Y_{1}\right) \otimes \widehat{C F D}\left(Y_{2}\right) \rightarrow \widehat{C F}\left(Y_{1} \cup_{F} Y_{2}\right)
$$

such that:

(1) $\Phi$ is a homotopy equivalence.

(2) Given generators $\mathbf{x}_{1} \otimes \mathbf{x}_{2}$ and $\mathbf{y}_{1} \otimes \mathbf{y}_{2}$ for $\widehat{C F A}\left(Y_{1}\right) \otimes \widehat{C F D}\left(Y_{2}\right), \mathfrak{s}\left(\Phi\left(\mathbf{x}_{1} \otimes \mathbf{x}_{2}\right)\right)=$ $\mathfrak{s}\left(\Phi\left(\mathbf{y}_{1} \otimes \mathbf{y}_{2}\right)\right)$ if and only if:

- $\mathfrak{s}\left(\mathbf{x}_{1}\right)=\mathfrak{s}\left(\mathbf{y}_{1}\right)=: \mathfrak{s}_{1}$

- $\mathfrak{s}\left(\mathbf{x}_{2}\right)=\mathfrak{s}\left(\mathbf{y}_{2}\right)=: \mathfrak{s}_{2}$, and

- the generators $g^{\prime}\left(\mathbf{x}_{1}\right) \times_{G^{\prime}} g^{\prime}\left(\mathbf{x}_{2}\right)$ and $g^{\prime}\left(\mathbf{y}_{1}\right) \times{ }_{G^{\prime}} g^{\prime}\left(\mathbf{y}_{2}\right)$ lie in the same $\mathbb{Z}$-orbit of $G_{A}^{\prime}\left(\mathcal{H}_{1}, \mathfrak{s}_{1}\right) \times_{G^{\prime}} G_{D}^{\prime}\left(\mathcal{H}_{2}, \mathfrak{s}_{2}\right)$. 
(3) If $\mathfrak{s}\left(\mathbf{x}_{1} \otimes \mathbf{x}_{2}\right)=\mathfrak{s}\left(\mathbf{y}_{1} \otimes \mathbf{y}_{2}\right)$ then

$$
g^{\prime}\left(\mathbf{y}_{1}\right) \times_{G^{\prime}} g^{\prime}\left(\mathbf{y}_{2}\right)=\lambda^{\operatorname{gr}\left(\Phi\left(\mathbf{x}_{1} \otimes \mathbf{x}_{2}\right), \Phi\left(\mathbf{y}_{1} \otimes \mathbf{y}_{2}\right)\right)} g^{\prime}\left(\mathbf{x}_{1}\right) \times_{G^{\prime}} g^{\prime}\left(\mathbf{x}_{2}\right) .
$$

For this paper, we will use a slightly larger grading group, and corresponding grading sets. Given a pointed matched circle $\mathcal{Z}=(Z, \mathbf{a}, M, z)$, let $G_{\mathbb{Q}}^{\prime}(\mathcal{Z})$ denote the $\mathbb{Q}$-central extension of $H_{1}(Z, \mathbf{a} ; \mathbb{Q})$ with multiplication given by

$$
\left(m_{1}, \alpha_{1}\right) \cdot\left(m_{2}, \alpha_{2}\right)=\left(m_{1}+m_{2}+m\left(\alpha_{2}, \partial \alpha_{1}\right), \alpha_{1}+\alpha_{2}\right),
$$

i.e., the same formula as Equation (2.7).

There is an obvious inclusion $G^{\prime} \rightarrow G_{\mathbb{Q}}^{\prime}$, so the $G^{\prime}$-grading on $\mathcal{A}(\mathcal{Z})$ induces a $G_{\mathbb{Q}^{\mathbb{S}}}^{\prime}$-grading on $\mathcal{A}(\mathcal{Z})$. Also, note that for $g \in G_{\mathbb{Q}}^{\prime}$ and $q \in \mathbb{Q}$ there is a well-defined element $q \cdot g \in G_{\mathbb{Q}}^{\prime}$ obtained by multiplying all of the coefficients in $g$ by $q$.

If $\mathcal{H}$ is a Heegaard diagram with $\partial \mathcal{H}=\mathcal{Z}$ (respectively $\partial \mathcal{H}=-\mathcal{Z}$ ), we can define a $G_{\mathbb{O}}^{\prime}$-grading on $\widehat{C F A}(\mathcal{H})$ (respectively $\widehat{C F D}(\mathcal{H})$ ) using Formula $(2.8)$. Given $\mathbf{x} \in \mathfrak{S}(\mathcal{H})$ let $P_{\mathbb{Q}}^{\prime}(\mathbf{x})$ denote the subgroup of $G_{\mathbb{Q}}^{\prime}$ generated by $\left\{q \cdot g^{\prime}(B) \mid B \in \pi_{2}(\mathbf{x}, \mathbf{x}), q \in \mathbb{Q}\right\}$. Fix a base generator $\mathbf{x}_{0} \in \mathfrak{S}(\mathcal{H}, \mathfrak{s})$. For any $\mathbf{x} \in \mathfrak{S}(\mathcal{H}, \mathfrak{s})$ choose a $B \in \pi_{2}\left(\mathbf{x}_{0}, \mathbf{x}\right)$ and define $\operatorname{gr}_{\mathbb{Q}}^{\prime}(\mathbf{x})=g^{\prime}(B)\left(\right.$ respectively $\left.\operatorname{gr}_{\mathbb{Q}}^{\prime}(\mathbf{x})=R\left(g^{\prime}(B)\right)\right)$, viewed as an element of $G_{A, \mathbb{Q}}^{\prime}(\mathcal{H}, \mathfrak{s}):=$ $P_{\mathbb{Q}}^{\prime}\left(\mathbf{x}_{0}\right) \backslash G_{\mathbb{Q}}^{\prime}(4 k)$ (respectively $G_{D, \mathbb{Q}}^{\prime}(\mathcal{H}, \mathfrak{s}):=G_{\mathbb{Q}}^{\prime}(4 k) / R\left(P_{\mathbb{Q}}^{\prime}\left(\mathbf{x}_{0}\right)\right)$ ).

There is also a refined grading on the algebra, by a group $G$ which is a $\mathbb{Z}$-central extension of $H_{1}(F(\mathcal{Z})$ ), and corresponding gradings on the modules; see [LOT08, Section 3.3] or [LOT10a, Section 3.1.1]. Generally we will work with the larger grading group in this paper, but see also Remark 3.3.

\section{From Bordered Floer to the Relative $\mathbb{Q}$-Grading}

Theorem 1. Suppose that $Y$ is a closed 3-manifold, decomposed along a connected surface as $Y=Y_{1} \cup_{F} Y_{2}$. Let $\mathcal{H}=\mathcal{H}_{1} \cup_{\mathcal{Z}} \mathcal{H}_{2}$ be a corresponding decomposition of a Heegaard diagram for $Y$. Suppose that $\mathbf{x}, \mathbf{y} \in \mathfrak{S}(\mathcal{H})$ are such that $\mathfrak{s}(\mathbf{x})$ and $\mathfrak{s}(\mathbf{y})$ are torsion, and $\left.\mathfrak{s}(\mathbf{x})\right|_{Y_{i}}=\left.\mathfrak{s}(\mathbf{y})\right|_{Y_{i}}=: \mathfrak{s}_{i}$ for $i=1,2$. Write $\mathbf{x}=\mathbf{x}_{1} \otimes \mathbf{x}_{2}$ and $\mathbf{y}=\mathbf{y}_{1} \otimes \mathbf{y}_{2}$, where $\mathbf{x}_{i}$ and $\mathbf{y}_{i}$ are in $\mathfrak{S}\left(\mathcal{H}_{i}\right)$. Then

(1) the generators $\operatorname{gr}_{\mathbb{Q}}^{\prime}\left(\mathbf{x}_{1}\right) \times_{G_{\mathbb{Q}}^{\prime}} \operatorname{gr}_{\mathbb{Q}}^{\prime}\left(\mathbf{x}_{2}\right)$ and $\operatorname{gr}_{\mathbb{Q}}^{\prime}\left(\mathbf{y}_{1}\right) \times_{G_{\mathbb{Q}}^{\prime}} \operatorname{gr}_{\mathbb{Q}}^{\prime}\left(\mathbf{y}_{2}\right)$ lie in the same $\mathbb{Q}$-orbit of $G_{A, \mathbb{Q}}^{\prime}\left(\mathcal{H}_{1}, \mathfrak{s}_{1}\right) \times_{G_{\mathbb{Q}}^{\prime}} G_{D, \mathbb{Q}}^{\prime}\left(\mathcal{H}_{2}, \mathfrak{s}_{2}\right)$ and

(2) $\operatorname{gr}_{\mathbb{Q}}^{\prime}\left(\mathbf{y}_{1}\right) \times_{G_{\mathbb{Q}}^{\prime}} \operatorname{gr}_{\mathbb{Q}}^{\prime}\left(\mathbf{y}_{2}\right)=\lambda^{\operatorname{gr}_{\mathbb{Q}}(\mathbf{x}, \mathbf{y})} \operatorname{gr}_{\mathbb{Q}}^{\prime}\left(\mathbf{x}_{1}\right) \otimes_{G_{\mathbb{Q}}^{\prime}} \operatorname{gr}_{\mathbb{Q}}^{\prime}\left(\mathbf{x}_{2}\right)$.

Proof. Since the statements are independent of the base generator used to define the grading sets for $\widehat{C F A}\left(\mathcal{H}_{1}, \mathfrak{s}_{1}\right)$ and $\widehat{C F D}\left(\mathcal{H}_{2}, \mathfrak{s}_{2}\right)$, we may choose $\mathbf{x}_{i}$ to be the base generator for $\mathcal{H}_{i}$.

Since $\mathfrak{s}(\mathbf{x})$ and $\mathfrak{s}(\mathbf{y})$ are torsion, it follows from [LL08] (cf. Section 2.1) that there is a rational domain $B \in \pi_{2}^{\mathbb{Q}}(\mathbf{x}, \mathbf{y})$ connecting $\mathbf{x}$ and $\mathbf{y}$. Intersecting $B$ with $\mathcal{H}_{1}$ and $\mathcal{H}_{2}$, we obtain rational domains $B_{i} \in \pi_{2}^{\mathbb{Q}}\left(\mathbf{x}_{i}, \mathbf{y}_{i}\right)$.

We argue that the rational domain $B_{i}$ can be used to compute the grading of $\mathbf{y}_{i}$ (which was originally defined using integral domains). Since $\mathfrak{s}\left(\mathbf{x}_{i}\right)=\mathfrak{s}\left(\mathbf{y}_{i}\right), \pi_{2}^{\mathbb{Q}}\left(\mathbf{x}_{i}, \mathbf{y}_{i}\right)=\pi_{2}\left(\mathbf{x}_{i}, \mathbf{y}_{i}\right) \otimes_{\mathbb{Z}} \mathbb{Q}$. That is, any rational domain $B_{i}$ connecting $\mathbf{x}_{i}$ and $\mathbf{y}_{i}$ can be written as

$$
B_{i}=q_{i, 1} C_{i, 1}+\cdots+q_{i, \ell} C_{i, \ell}
$$

where the $q_{i, j} \in \mathbb{Q}$ and the $C_{i, j} \in \pi_{2}\left(\mathbf{x}_{i}, \mathbf{y}_{i}\right)$. (To see this, note that $\pi_{2}\left(\mathbf{x}_{i}, \mathbf{y}_{i}\right)$ is an affine copy of $H_{2}\left(Y_{i}, \partial Y_{i} ; \mathbb{Z}\right)$ while $\pi_{2}^{\mathbb{Q}}\left(\mathbf{x}_{i}, \mathbf{y}_{i}\right)$ is an affine copy of $H_{2}\left(Y_{i}, \partial Y_{i} ; \mathbb{Q}\right)$.) Consequently, $B_{i}$ differs from any integral domain in $\pi_{2}\left(\mathbf{x}_{i}, \mathbf{y}_{i}\right)$ by a rational periodic domain, and hence has 
the same image in $G_{A, \mathbb{Q}}^{\prime}\left(\mathcal{H}_{1}, \mathfrak{s}_{1}\right)$ or $G_{D, \mathbb{Q}}^{\prime}\left(\mathcal{H}_{2}, \mathfrak{s}_{2}\right)$. In formulas, as elements of $G_{A, \mathbb{Q}}^{\prime}\left(\mathcal{H}_{1}, \mathfrak{s}_{1}\right)$ and $G_{D, \mathbb{Q}}^{\prime}\left(\mathcal{H}_{2}, \mathfrak{s}_{2}\right)$ respectively,

$$
g^{\prime}\left(B_{1}\right)=\left(-e\left(B_{1}\right)-n_{\mathbf{x}_{1}}\left(B_{1}\right)-n_{\mathbf{y}_{1}}\left(B_{1}\right), \partial^{\partial} B_{1}\right)=\operatorname{gr}^{\prime}\left(\mathbf{y}_{1}\right)
$$

and

$$
R\left(g^{\prime}\left(B_{2}\right)\right)=\left(-e\left(B_{2}\right)-n_{\mathbf{x}_{2}}\left(B_{2}\right)-n_{\mathbf{y}_{2}}\left(B_{2}\right), r_{*}\left(\partial^{\partial} B_{2}\right)\right)=\operatorname{gr}^{\prime}\left(\mathbf{y}_{2}\right) .
$$

Note also that $\partial^{\partial} B_{2}=-\partial^{\partial} B_{1}$.

Thus, with our choice of base generator, $\operatorname{gr}_{\mathbb{Q}}^{\prime}\left(\mathbf{x}_{1}\right) \times_{G_{\mathbb{Q}}^{\prime}} \operatorname{gr}_{\mathbb{Q}}^{\prime}\left(\mathbf{x}_{2}\right)=0$ while

$$
\begin{aligned}
\operatorname{gr}_{\mathbb{Q}}^{\prime}\left(\mathbf{y}_{1}\right) \times_{G_{\mathbb{Q}}^{\prime}} \operatorname{gr}_{\mathbb{Q}}^{\prime}\left(\mathbf{y}_{2}\right) & =\left(-e\left(B_{1}\right)-n_{\mathbf{x}_{1}}\left(B_{1}\right)-n_{\mathbf{y}_{1}}\left(B_{1}\right)-e\left(B_{2}\right)-n_{\mathbf{x}_{2}}\left(B_{2}\right)-n_{\mathbf{y}_{2}}\left(B_{2}\right), 0\right) \\
& =\left(-e(B)-n_{\mathbf{x}}(B)-n_{\mathbf{y}}(B), 0\right)=\lambda^{\operatorname{gr}_{\mathbb{Q}}}(\mathbf{x}, \mathbf{y})
\end{aligned}
$$

as desired.

To complete the computation of the relative $\mathbb{Q}$-grading on $\widehat{C F}$, we observe that it is always possible to find a splitting satisfying the conditions of Theorem 1.

Lemma 3.1. Given any 3-manifold $Y$ and torsion $\operatorname{spin}^{c}$-structures $\mathfrak{s}$ and $\mathfrak{s}^{\prime}$ on $Y$ there is a decomposition $Y=Y_{1} \cup_{F} Y_{2}$ of $Y$ along a connected surface $F$ so that $\left.\mathfrak{s}\right|_{Y_{i}}=\left.\mathfrak{s}^{\prime}\right|_{Y_{i}}$ for $i=1,2$.

Proof. Since a handlebody has a unique $\operatorname{spin}^{c}$-structure, any Heegaard decomposition for $Y$ satisfies the conditions.

Corollary 3.2. The $G^{\prime}$-set grading $\mathrm{gr}^{\prime}$ defined in [LOT08] determines the relative $\mathbb{Q}$-grading on $\widehat{H F}$.

Proof. By definition, the grading gr' determines $\operatorname{gr}_{\mathbb{Q}}^{\prime}$ which in turn, by Lemma 3.1 and Theorem 1 , determines the relative $\mathbb{Q}$ grading.

Remark 3.3. It is sometimes convenient to work with the smaller grading group $G$ from [LOT08], rather than $G^{\prime}$. To obtain a $G$-set grading on $\widehat{C F D}$ and $\widehat{C F A}$, one conjugates by grading refinement data; see [LOT10a, Section 3.1.1]. In the proof of Theorem 1, since one works with the same grading refinement data on the two sides, it cancels out in the computation. Thus, Theorem 1 holds with respect to the small grading group, as well.

Remark 3.4. In [LOT10b], we give an algorithm for computing $\widehat{H F}(Y)$ by taking a Heegaard decomposition of $Y$ and factoring the gluing map into arc-slides. For such a decomposition, the hypotheses of Theorem 1 are automatically satisfied. Thus, keeping track of the $G_{\mathbb{Q}^{-}}^{\prime}$ gradings along the way, [LOT10b] automatically computes the relative $\mathbb{Q}$-grading on $\widehat{H F}(Y)$.

Remark 3.5. Instead of defining a $G_{\mathbb{Q}^{-}}^{\prime}$ grading on $\widehat{C F D}$ by (roughly) tensoring $G^{\prime}$-grading with $\mathbb{Q}$ as above, we could instead use rational domains to induce a $G^{\prime}$-grading. The resulting relative grading agrees with the one above when the one above is defined, but it is defined more often. Theorem 1 then no longer needs the hypothesis that $\left.\mathfrak{s}(\mathbf{x})\right|_{Y_{i}}=\left.\mathfrak{s}(\mathbf{y})\right|_{Y_{i}}$. The drawback is that, for this definition, $\mathrm{gr}_{\mathbb{Q}}^{\prime}$ is no longer induced from $\mathrm{gr}^{\prime}$, so one would not obtain Corollary 3.2. 


\section{EXAMPLES}

We give an application of Theorem 1 to computing the $\mathbb{Q}$-graded Heegaard Floer homology groups of surgeries on some knots in $S^{3}$. Our knots are rather simple (the unknot and the trefoil), and hence the graded Heegaard Floer homology groups on their surgeries have been known for some time; but these computations do give a nice illustration of the theorem.

To start, let $Y$ denote the (-2)-framed complement of the left-handed trefoil $T$. By [LOT08, Theorem 11.7], $\widehat{C F D}(Y)$ is given by

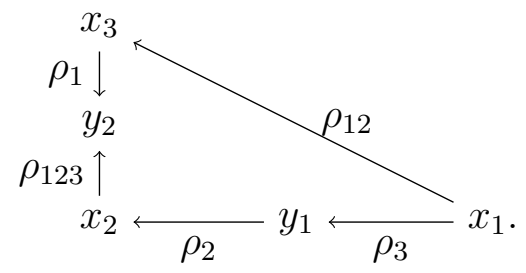

If we take $x_{3}$ as the base generator then the gradings lie in $G^{\prime} /\langle(-3 / 2 ;-1,1,2)\rangle$, and are given by:

$$
\begin{aligned}
& \operatorname{gr}\left(x_{1}\right)=(1 ; 0,2,2) /\langle(-3 / 2 ;-1,1,2)\rangle \\
& \operatorname{gr}\left(x_{2}\right)=(1 / 2 ; 0,1,1) /\langle(-3 / 2 ;-1,1,2)\rangle \\
& \operatorname{gr}\left(x_{3}\right)=(0 ; 0,0,0) /\langle(-3 / 2 ;-1,1,2)\rangle \\
& \operatorname{gr}\left(y_{1}\right)=(3 / 2 ; 0,2,1) /\langle(-3 / 2 ;-1,1,2)\rangle \\
& \operatorname{gr}\left(y_{2}\right)=(-1 / 2 ;-1,0,0) /\langle(-3 / 2 ;-1,1,2)\rangle
\end{aligned}
$$

(compare [LOT08, Section 10.9]).

Let $\mathcal{H}_{0}$ denote the $\infty$-framed solid torus. Then $\widehat{C F A}\left(\mathcal{H}_{0}\right)$ has one generator $n$ with $m_{3}\left(n, \rho_{2}, \rho_{1}\right)=n$. In particular,

$$
\operatorname{gr}(n)=\operatorname{gr}(n) \operatorname{gr}\left(\rho_{2}\right) \operatorname{gr}\left(\rho_{1}\right) \lambda=\operatorname{gr}(n)(-1 / 2 ; 0,1,0)(-1 / 2 ; 1,0,0)=\operatorname{gr}(n)(-1 / 2 ; 1,1,0) .
$$

So, $\operatorname{gr}(n)$ lies in $\langle(-1 / 2 ; 1,1,0)\rangle \backslash G^{\prime}$.

Tensoring the two together, we find that $\widehat{C F A}\left(\mathcal{H}_{0}\right) \otimes \widehat{C F D}(Y)$ is generated by $n \otimes y_{1}$ and $n \otimes y_{2}$, with no differential. It follows at once that $\widehat{H F}\left(S_{-2}^{3}(T)\right) \cong \mathbb{F}_{2} \oplus \mathbb{F}_{2}$, i.e. $S_{-2}^{3}(T)$ has the same (ungraded) Heegaard Floer homology as a lens space; this was, of course, known before [OS05].

So far, we have found that the ungraded Heegaard Floer homology of -2 surgery on the trefoil and the unknot are the same. They are, however, distinguished by their relative $\mathbb{Q}$-gradings, which we can recover from the bordered invariants, as follows.

The computation above gives

$$
\begin{aligned}
& \operatorname{gr}\left(n \otimes y_{1}\right)=\langle(-1 / 2 ; 1,1,0)\rangle \backslash(3 / 2 ; 0,2,1) /\langle(-3 / 2 ;-1,1,2)\rangle \\
& \operatorname{gr}\left(n \otimes y_{2}\right)=\langle(-1 / 2 ; 1,1,0)\rangle \backslash(-1 / 2 ;-1,0,0) /\langle(-3 / 2 ;-1,1,2)\rangle .
\end{aligned}
$$

Working in $G_{\mathbb{Q}}^{\prime}$, we can rewrite the first of these equations as:

$$
\begin{aligned}
& \operatorname{gr}\left(n \otimes y_{1}\right)=\langle(-1 / 2 ; 1,1,0)\rangle \backslash(3 / 4 ;-3 / 2,-3 / 2,0) \cdot(3 / 2 ; 0,2,1) \\
& \cdot(3 / 4 ; 1 / 2,-1 / 2,-1) /\langle(-3 / 2 ;-1,1,2)\rangle \\
&=\langle(-1 / 2 ; 1,1,0)\rangle \backslash(1 ;-1,0,0) /\langle(-3 / 2 ;-1,1,2)\rangle .
\end{aligned}
$$


Consequently, the grading difference between $n \otimes y_{1}$ and $n \otimes y_{2}$ is $3 / 2$.

By contrast, the invariant of the -2-framed unknot has three generators:

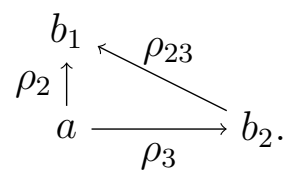

If we take $a$ as the base generator, the gradings lie in $G^{\prime} /\langle(1 / 2 ;-1,1,2)\rangle$, and are given by:

$$
\begin{aligned}
\operatorname{gr}^{\prime}(a) & =(0 ; 0,0) /\langle(1 / 2 ;-1,1,2)\rangle \\
\operatorname{gr}^{\prime}\left(b_{1}\right) & =(-1 / 2 ; 0,1,0) /\langle(1 / 2 ;-1,1,2)\rangle \\
\operatorname{gr}^{\prime}\left(b_{2}\right) & =(-1 / 2 ; 0,0,-1) /\langle(1 / 2 ;-1,1,2)\rangle .
\end{aligned}
$$

This gives

$$
\begin{aligned}
& \operatorname{gr}^{\prime}\left(n \otimes b_{1}\right)=\langle(-1 / 2 ; 1,1,0)\rangle \backslash(-1 / 2 ; 0,1,0) /\langle(1 / 2 ;-1,1,2)\rangle \\
& \operatorname{gr}^{\prime}\left(n \otimes b_{2}\right)=\langle(-1 / 2 ; 1,1,0)\rangle \backslash(-1 / 2 ; 0,0,-1) /\langle(1 / 2 ;-1,1,2)\rangle .
\end{aligned}
$$

Working in $G_{\mathbb{Q}}^{\prime}$, we can rewrite the second of these equations as:

$$
\operatorname{gr}^{\prime}\left(n \otimes b_{1}\right)=\langle(-1 / 2 ; 1,1,0)\rangle \backslash(0 ; 0,0,-1) /\langle(1 / 2 ;-1,1,2)\rangle .
$$

Consequently, the grading difference between $n \otimes b_{1}$ and $n \otimes b_{2}$ is $-1 / 2$. Thus we see that the relative $\mathbb{Q}$-grading distinguishes the Heegaard Floer homology of -2 surgery on the trefoil from -2 surgery on the unknot.

\section{REFERENCES}

[Frø04] Kim A. Frøyshov, An inequality for the h-invariant in instanton Floer theory, Topology 43 (2004), no. 2, 407-432.

[Gre] Joshua Evan Greene, Lattices, graphs, and Conway mutation, arXiv:1103.0487.

[Lip06] Robert Lipshitz, A cylindrical reformulation of Heegaard Floer homology, Geom. Topol. 10 (2006), 955-1097, arXiv:math.SG/0502404.

[LL08] Dan A. Lee and Robert Lipshitz, Covering spaces and $\mathbb{Q}$-gradings on Heegaard Floer homology, J. Symplectic Geom. 6 (2008), no. 1, 33-59.

[LOT08] Robert Lipshitz, Peter S. Ozsváth, and Dylan P. Thurston, Bordered Heegaard Floer homology: Invariance and pairing, 2008, arXiv:0810.0687v4.

[LOT10a] _ Bimodules in bordered Heegaard Floer homology, 2010, arXiv:1003.0598v3.

[LOT10b] _ Computing $\widehat{H F}$ by factoring mapping classes, 2010, arXiv:1010.2550v3.

[OS05] Peter Ozsváth and Zoltán Szabó, On knot Floer homology and lens space surgeries, Topology 44 (2005), no. 6, 1281-1300.

[OSz04] Peter S. Ozsváth and Zoltán Szabó, Holomorphic disks and topological invariants for closed threemanifolds, Ann. of Math. (2) 159 (2004), no. 3, 1027-1158, arXiv:math.SG/0101206.

[OSz06]_ Holomorphic triangles and invariants for smooth four-manifolds, Adv. Math. 202 (2006), no. 2, 326-400, arXiv:math.SG/0110169.

[Sar10] Sucharit Sarkar, Maslov index formulas for Whitney n-gons, 2010, arXiv:math/0609673v3.

[SW10] Sucharit Sarkar and Jiajun Wang, An algorithm for computing some Heegaard Floer homologies, Ann. of Math. (2) 171 (2010), no. 2, 1213-1236, arXiv:math/0607777. 
Department of Mathematics, Columbia University, New York, NY 10027

E-mail address: lipshitz@math.columbia.edu

Department of Mathematics, Princeton University, New Jersey, 08544

E-mail address: petero@math.princeton.edu

Department of Mathematics, UC Berkeley, 970 evans Hall, Berkeley, CA 94720

E-mail address: dpt@math. berkeley.edu 